\title{
Association of the PGC-1 $\alpha$ rs 8192678 variant with microalbuminuria in subjects with type 2 diabetes mellitus
}

\author{
Sarah L. Prior ${ }^{a, *}$, Amy R. Clark ${ }^{\mathrm{a}}$, Danielle A. Jones ${ }^{\mathrm{a}}$, Steve C. Bain ${ }^{\mathrm{a}}$, Steve J. Hurel ${ }^{\mathrm{b}}$, \\ Steve E. Humphries ${ }^{\mathrm{c}}$ and Jeffrey W. Stephens ${ }^{\mathrm{a}}$ \\ ${ }^{a}$ Diabetes Research Group, Institute of Life Science, Swansea University, Swansea, UK \\ ${ }^{\mathrm{b}}$ Department of Diabetes and Endocrinology, UCL Hospitals, London, UK \\ ${ }^{\mathrm{c}}$ Centre for Cardiovascular Genetics, British Heart Foundation Laboratories, Royal Free and University College \\ London Medical School, London, UK
}

\begin{abstract}
PPAR- $\gamma$ co-activator- $1 \alpha$ (PGC-1 $\alpha)$ is a tissue-specific transcriptional co-activator involved in the regulation of antioxidant enzymes. The A-allele of the $r s 8192678$ PGC-1 $\alpha(\mathrm{G}>\mathrm{A})$ gene variant has previously been associated with nephropathy in Korean and Indian-Asian type 2 diabetes mellitus (T2DM) samples. Our aim was to examine the association between this variant and urine albumin exccretion in European subjects with T2DM.

Genotyping was performed on 583 European subjects with T2DM and examined in relation to urinary albumin, plasma oxidizedLDL and small dense-LDL percentage. We observed a significant association between genotype (GG/GA/AA) and urinary albumin (normoalbuminuria $\vee$ micro/macroalbuminuria: 48.6/39.7/11.7\% v 38.2/51.2/10.5\%, $p=0.02$; for GG $\vee$ GA/AA, $p=$ 0.01). The odds ratio for micro/macroalbuminuria in GA and AA subjects relative to GG were 1.70 [1.15-2.50], $p=0.008$ and 1.20 [0.66-2.16], $p=0.56$ respectively (for GA/AA v GG: 1.58 [95\%CI: 1.09-2.27], $p=0.02$ ). There was a significant association between the A allele and a higher percentage of small dense-LDL particles (GG v GA v AA: 70.8 [58.01-81.06] \% v $72.8[56.18-81.19] \%$ v $78.9[67.16-85.33] \%, p=0.03)$.

In European subjects with T2DM the GA relative to the GG genotype is associated with a $70 \%$ increase in the risk of micro/microalbuminuria. Furthermore, homozygosity for the A-allele is also associated with a preponderance of small dense-LDL particles.
\end{abstract}

Keywords: PGC-1 $\alpha$, type 2 diabetes mellitus, microalbuminuria, oxidative stress

\section{Introduction}

The endothelium plays a major role in vascular homeostasis regulating vascular tone, platelet activity, leukocyte adhesion, inflammation and thrombosis [1, 2]. Endothelial dysfunction is associated with cardiovascular disease (CVD), diabetes mellitus, and chronic renal impairment [3]. In subjects with diabetes, endothelial dysfunction is associated with microalbumin-

* Corresponding author: Dr. Sarah Prior, Diabetes Research Group, 3rd Floor, Institute of Life Sciences, Swansea University, Swansea, SA2 8PP, UK. Tel.: +44 01792 295073; Fax: +44 01792 602147; E-mail: s.l.prior@swansea.ac.uk uria and proteinuria [4], and therefore microalbuminuria may be regarded as a marker of vascular dysfunction and CVD risk in subjects [5]. Microalbuminuria is also associated with increased oxidative stress and elevated inflammatory cytokines such as interleukin-1, interleukin-6 and TNF- $\alpha$ [6]. CVD risk in diabetes increases with progression from normoalbimonuria to microalbuminuria and macroalbuminuria [5]. Compared to subjects with normoalbuminura survival for subjects with microalbuminuria is reduced by $20 \%$ and for those with macroalbuminuria by $30 \%$ [7].

Peroxisome proliferator-activated receptor- $\gamma$ coactivator- $1 \alpha(\mathrm{PGC}-1 \alpha)$ is a tissue-specific transcriptional co-activator [8] involved in the activation of sev- 
eral nuclear hormone receptors essential for the regulation of adaptive thermogenesis, cellular respiration, adipogenesis and oxidative metabolism [9]. Of particular importance, PGC- $1 \alpha$ is involved in the regulation of genes involved in glucose and lipid metabolism [10] as well as antioxidant enzymes such as glutathione peroxidase-1 and superoxide dismutase-2 [11]. The $P G C$ - $1 \alpha$ gene is located on chromosome 4p15.1 [12] and spans 13 exons [13]. The PGC- $1 \alpha$ protein stimulates mitochondrial biogenesis and respiration by coactivating nuclear respiratory factor- 1 , which in turn binds to the transcription factor peroxisome proliferatoractivated receptor- $\gamma$ and induces the expression of mitochondrial uncoupling protein-2 $[8,14]$ which protects against cellular oxidative damage [15-17].

Genetic variation at position 1444 in exon 8 of the $P G C$ - $1 \alpha$ gene $(r s 8192678)$ results in a $\mathrm{G}>\mathrm{A}$ base substitution [18], causing a glycine to serine change in the amino acid sequence (Gly482Ser) and a structural protein change [19]. The minor A-allele (coding a serine residue) results in lower protein levels and reduced activity of the PCG- $1 \alpha$ protein in muscle tissue from subjects with insulin-resistance and diabetes [20,21] and is also associated with lower levels of fatty acid oxidation [22]. Carriers of the A-allele also exhibit increased levels of DNA damage [11]. The AA genotype (Ser/Ser) has been associated with hypertension in male subjects with type 2 diabetes mellitus (T2DM) [18] and furthermore is also associated with an increased risk of T2DM [23]. Two previous studies have investigated this gene variant with respect to diabetic nephropathy. In an Asian Indian sample, the A-allele had an autosomal dominant association with diabetic nephropathy and homozygosity for the A-allele was associated with an eight-fold increase in risk [24]. With a Korean sample, the A-allele was associated with a 1.5-fold increase in nephropathy risk [25].

Our aim was to examine the association between the $r s 8192678$ (Gly482Ser) $P G C-1 \alpha$ gene variant with urinary albumin as a marker of endothelial dysfunction, in a cross-sectional sample of European subjects with T2DM.

\section{Methods}

\subsection{Subjects}

Ethical approval was obtained from the UCL and UCL Hospitals Research Ethics Committee and all subjects gave written informed consent before recruit- ment. Subjects were recruited as part of the University College London Diabetes and Cardiovascular Study (UDACS), described elsewhere [17,26]. Briefly, this comprises of consecutive subjects recruited from the diabetes clinic at University College London Hospitals (UCLH) NHS Trust between the years 2001-2002. All subjects had diabetes according to WHO criteria [27]. Microalbuminuria was defined as an albumin:creatinine ratio (ACR) of greater than $2.5 \mathrm{mg} / \mathrm{mmol}$ in men and $3.5 \mathrm{mg} / \mathrm{mmol}$ in women [28-30]. Macroalbuminuria was defined as an ACR $>30 \mathrm{mg} / \mathrm{mmol}$. At the outset subjects with micro/macroalbuminuria were grouped together for analysis. All urine samples were firstvoided. None of the subjects were knowingly taking any form of vitamin supplementation. Plasma samples were collected during the twelve months of recruitment and stored immediately at $-80^{\circ} \mathrm{C}$. Samples were collected during routine diabetes clinic visit. None of the patients were fasting at the time of collection.

\subsection{Genotyping for the rs8192678 gene variant}

Genomic DNA was extracted from 5ml EDTA blood samples. Genotyping was conducted using polymerase chain reaction amplification followed by restriction digest (HpaII). Primer sequences were: forward TG CTACCTGAGAGAGACTTTG and reverse CTTTCA TCTTCGCTGTCATC [20]. Genotype was confirmed by two independent technicians and any discrepancy resolved by repeat genotyping.

\subsection{Measurement of plasma total anti-oxidant status}

Plasma total anti-oxidant status (TAOS) was measured by Sampson's modification of Laight's photometric microassay [31], using $2.5 \mu \mathrm{l}$ citrated plasma samples in 96-well ELISA plates. The TAOS of plasma was determined by its capacity to inhibit the peroxidase-mediated formation of the 2,2-azino-bis-3ethylbensthiazoline-6-sulfonic acid (ABTS+) radical The inter- and intra-assay coefficients of variation were $10.1 \%$ and $4.3 \%$ respectively. Previously, we have shown that baseline plasma TAOS is associated with prospective risk and has a good correlation with plasma $\mathrm{F}_{2}$-isoprostanes [32].

\subsection{Measurement of plasma $O x-L D L$}

Plasma oxidized LDL (Ox-LDL) was measured using a commercially available Enzyme-Linked Im- 
munosorbent Assay (ELISA) kit supplied by Mercodia (Uppsala, Sweden). In this assay a monoclonal antibody is directed against antigenic determinants in the Ox-LDL molecule (mAB-4E6).

\subsection{Measurement of low density lipoprotein particle diameter}

Low density lipoprotein (LDL) at $d 1.019-1.063 \mathrm{~g} / \mathrm{ml}$ was isolated from EDTA plasma. Electrophoresis was performed as previously described [33]. Polyacrylamide gels were used to determine LDL peak particle diameter (LDL-PPD) and LDL mean particle diameter (LDL-MPD) and the \% SD-LDL was determined from the LDL particle size [34].

\subsection{Measurement of plasma C-reactive protein}

C-reactive protein (CRP) levels were measured with a highly sensitive in-house ELISA assay with rabbit anti-CRP (Dako, Copenhagen, Denmark) as a catching and tagging antibody, as described previously [35]. The inter-assay and intra-assay CV were $8 \%$ and $10 \%$ respectively and limit of detection $0.15 \mathrm{mg} / \mathrm{L}$.

\subsection{Statistical analysis}

Statistical analysis was performed using SPSS (version 10.1, SPSS Inc., Chicago). Data are reported for those individuals with available genotype. Results are presented as mean and standard deviation. Deviations from Hardy-Weinberg equilibrium were considered using chi-squared tests. Allele frequencies are shown with the $95 \%$ confidence interval. The association between genotype and urinary albumin excretion was examined by chi-squared testing and by calculating the odds ratios (ORs) with $95 \%$ confidence intervals derived by logistic regression analysis. For continuous data that was normally distributed after log transformation, the geometric mean and approximate standard deviation is shown. This included systolic blood pressure, diastolic blood pressure, body mass index (BMI), $\mathrm{HBA}_{1 \mathrm{c}}$, random glucose, LDL-cholesterol and triglyceride. For duration of diabetes, creatinine, HDL-cholesterol, TAOS, Ox-LDL and \% small dense LDL particles, the data could not be transformed to a normal distribution and so the data is shown as median and interquartile range. Analysis of variance (ANOVA) was used to assess the association between genotype and baseline characteristics for data that was normally distributed after log transformation. For duration of diabetes, creatinine, HDL-cholesterol, TAOS, Ox-LDL and $\%$ small dense LDL particles, data was analysed by the Kruskal-Wallis test and Mann-Whitney tests. Chisquared tests were used to compare differences in categorical variables by genotype. In all cases a p-value of less than 0.05 was considered statistically significant. Two sided statistical testing was performed.

\section{Results}

\subsection{Characteristics of study subjects}

As shown in Table 1, micro/macroalbuminuria was associated with the male gender, increasing age, systolic blood pressure, serum creatinine, $\mathrm{HbA}_{1 c}$ and lower HDL-cholesterol. There were also a higher proportion of ACE inhibitor and aspirin use in those with micro/macroalbuminuria.

\subsection{Genotype data}

Genotype data was available on 980/1002 (97.8\%) subjects. We chose to focus on European patients with T2DM since all previous published studies looking at this variant have focussed on T2DM. There were a total of 583 European subjects with T2DM with available genotype and data on urine protein excretion was available on 575 subjects $(98.6 \%)$. The genotype distribution for the $r s 8192678(1444 G>A)$ gene variant was in Hardy-Weinberg equilibrium (GG/GA/AA: $254 / 264 / 65, \chi^{2}=0.08, p=0.77$ ), with a minor Aallele frequency of 0.34 [95\%CI: $0.31-0.37$ ]. The mean age of the GG subjects was approximately two years older than the A-allele carriers (GG/GA/AA: 68.0/65.4/66.4 years, $p=0.03$ ). Furthermore, there were a significantly lower proportion of males with the GG genotype (GG/GA/AA: $53.5 / 65.9 / 56.9 \%$ males, $p=0.02)$.

\subsection{Genotype association with urinary protein excretion}

There was a significant difference in genotype distribution in the urinary albumin excretion groups (GG/ GA/AA within normoalbuminuria $\mathrm{v}$ micro/macroalbuminuria: $48.6 / 39.7 / 11.7 \%$ v 38.2/51.2/10.6\%, $p=$ 0.02 ; for GG v GA/AA, $p=0.02$ ) (Table 1). Relative to the GG genotype the OR for micro/macroalbuminuria in GA and AA subjects were 1.64 [1.12-2.33], $p=$ 0.005 and 1.14 [0.66-1.98], $p=0.64$ respectively. The 
Table 1

Baseline differences by urine protein excretion

\begin{tabular}{|c|c|c|c|}
\hline Variable & $\begin{array}{l}\text { Normoalbuminuria } \\
\quad(N=290)\end{array}$ & $\begin{array}{l}\text { Micro/macroalbuminuria } \\
\qquad(N=285)\end{array}$ & $\mathrm{P}$ \\
\hline Age (years) & $65.6(10.7)$ & $67.8(11.2)$ & 0.01 \\
\hline Males \% (n) & 52.9 & 66.4 & 0.001 \\
\hline Duration of diabetes (years) $\#$ & $8[4-15]$ & $10[5-17]$ & 1.00 \\
\hline Systolic blood pressure $(\mathrm{mmHg})^{*}$ & $138(19)$ & $143(22)$ & 0.006 \\
\hline Diastolic blood pressure $(\mathrm{mmHg})^{*}$ & $79(11)$ & $80(12)$ & 0.21 \\
\hline Body mass index $\left(\mathrm{kg} / \mathrm{m}^{2}\right)^{*}$ & $29.4(5.7)$ & $39.3(5.3)$ & 0.79 \\
\hline Creatinine $(\mu \mathrm{mol} / \mathrm{L})^{\#}$ & $87[77-100]$ & $96[82-120]$ & $<0.001$ \\
\hline $\operatorname{HbA} 1 \mathrm{c}(\%)^{*}$ & $7.5(1.5)$ & $7.8(1.8)$ & 0.006 \\
\hline Random glucose $\mathrm{mmol} / \mathrm{L})^{*}$ & $9.3(4.0)$ & $10.6(4.6)$ & $<0.001$ \\
\hline LDL cholesterol $(\mathrm{mmol} / \mathrm{L})^{*}$ & $2.6(0.4)$ & $2.6(0.4)$ & 0.89 \\
\hline HDL cholesterol $(\mathrm{mmol} / \mathrm{L})^{\#}$ & $1.3[1.1-1.6]$ & $1.2[1.0-1.5]$ & 0.002 \\
\hline Triglyceride $(\mathrm{mmol} / \mathrm{L})^{*}$ & $1.9(1.1)$ & $1.9(1.1)$ & 0.80 \\
\hline TAOS $(\%)^{\#}$ & $44.8[36.9-52.1]$ & $43.8[33.7-52.0]$ & 0.27 \\
\hline Ox-LDL $\#$ & $45.8[34.8-54.3]$ & $48.6[38.1-62.2]$ & 0.01 \\
\hline Mean LDL size (nm) & $26.62(0.88)$ & $26.50(0.96)$ & 0.29 \\
\hline Peak particle diameter LDL (nm) & $26.82(0.85)$ & $26.73(0.80)$ & 0.21 \\
\hline$\%$ Small dense-LDL \# & $72.8[60.0-81.5]$ & $72.2[55.2-81.3]$ & 0.36 \\
\hline Current smoker \% (n) & $17.0 \%(49)$ & $13.9 \%(39)$ & 0.31 \\
\hline ACE inhibitor use \% (n) & $38.4 \%(111)$ & $61.1 \%(174)$ & $<0.001$ \\
\hline Aspirin use \% (n) & $44.3 \%(128)$ & $57.6 \%(164)$ & 0.001 \\
\hline Insulin use \% (n) & $26.0 \%(75)$ & $26.8 \%(76)$ & 0.85 \\
\hline Statin use $\%(\mathrm{n})$ & $30.8 \%(89)$ & $33.1 \%(94)$ & 0.56 \\
\hline Genotype (GG/GA/AA) \% (n) & $\begin{array}{c}48.6 / 39.7 / 11.7 \\
(141 / 115 / 34)\end{array}$ & $\begin{array}{c}38.2 / 51.2 / 10.6 \\
(109 / 146 / 30)\end{array}$ & 0.02 \\
\hline
\end{tabular}

Urine albumin data was available on 575 of the 583 with genotype data $(98.6 \%)$. \# Median and interquartile range shown for HDL, TAOS and Ox-LDL. * Log transformed data. Mean and standard deviation shown or geometric mean and approximate standard deviation for log transformed data. Analysis performed by ANOVA after appropriate transformation of non-normally distributed data and by Kruskal-Wallis for HDL, TAOS and Ox-LDL. $\chi^{2}$-test was used to compare groups.

OR for micro/macroalbuminuria in the GA/AA subjects relative to GG subjects was 1.53 [95\%CI: 1.10-2.13], $p=0.01$. After adjusting for the variables associated with urine albumin excretion described in section 3.1 (male gender, age, systolic blood pressure, serum creatinine, $\mathrm{HbA}_{1 c}$, lower HDL-cholesterol, ACE inhibitor and aspirin use) the association remained significant with the OR for micro/macroalbuminuria relative to GG in GA and AA subjects were 1.70 [1.15$2.50], p=0.008$ and 1.20 [0.66-2.16], $p=0.56$ respectively, with the OR for micro/macroalbuminuria in the GA/AA subjects relative to GG subjects being 1.58 [95\%CI: 1.09-2.27], $p=0.02$.

\subsection{Association with markers of oxidative damage and small dense- $L D L$}

Plasma Ox-LDL was higher in those with micro/macroalbuminuria compared to those with normoalbuminuria (48.6 [38.1-62.2]U/L v 45.8 [34.8$54.3] \mathrm{U} / \mathrm{L}$ respectively, $p=0.01$ ). No association was observed between urinary albumin excretion and plasma TAOS (43.8 [33.7-52.0]\% v 44.8 [36.9-52.1]\%, $p=0.27)$ or LDL particle size (MPD: $26.73(0.80) \mathrm{nm}$ v $26.82(0.85) \mathrm{nm}, p=0.21)$.

No association between genotype (GG/GA/AA) and plasma TAOS (45.4 [36.9-51.8]\% v 43.0 [33.9-52.8]\% v 44.9 [35.0-52.8]\%, $p=0.43)$, Ox-LDL (46.1 [35.2$58.7]$ v 49.0 [39.1-57.9] v 43.4 [32.4-56.0], $p=0.13)$ or LDL -MPD (26.77 (0.71)nm v $26.72(0.91) n m$ v $27.02(0.84) \mathrm{nm}, p=0.07)$ was observed. There was a significant association between the AA genotype the percentage of small dense-LDL particles (GG v GA v AA: 70.8 [58.01-81.06]\% v 72.8 [56.18-81.19]\% v 78.9 [67.16-85.33]\%, $p=0.03)$.

\section{Discussion}

This study examined the $r s 8192678(1444 \mathrm{G}>\mathrm{A})$ variant of the $P G C-1 \alpha$ gene in relation to micro/macroalbuminuria in a European sample of subjects with T2DM. We chose from the outset to focus on European subjects because of differences in genotype distribution by ethnic backgrounds (Table 2). Previously a higher prevalence of the GG genotype has been 
Table 2

Genotype distribution grouped by ethnic origin

\begin{tabular}{lllcc}
\hline Ethnic Group & \multicolumn{1}{c}{ GG } & \multicolumn{1}{c}{ GA } & AA & A-allele frequency \\
\hline African & $79.2 \%(61)$ & $18.2 \%(14)$ & $2.6 \%(2)$ & $0.12[0.07-0.17]$ \\
European & $44.5 \%(345)$ & $44.4 \%(344)$ & $11.1 \%(86)$ & $0.33[0.31-0.36]$ \\
Indian-Asian & $54.5 \%(60)$ & $38.2 \%(42)$ & $7.3 \%(8)$ & $0.26[0.21-0.32]$ \\
Asian & $22.2 \%(4)$ & $61.1 \%(11)$ & $16.7 \%(3)$ & $0.66[0.54-0.78]$ \\
\hline
\end{tabular}

Genotype data was available on 980/1002 (97.8\%). Genotype distribution (\% and $\mathrm{n}$ within each ethnic group) is shown. Analysis between groups $\chi^{2}=41.2, p<$ 0.000001 .

observed in Africans and of the AA genotype in Asians (http://www.ncbi.nlm.nih.gov/projects/SNP/snp_ref. cgi?rs=8192678). As expected we observed that subjects with micro/macroalbuminuria had increased plasma levels of Ox-LDL, a finding that was independent of other risk factors. We also observed that the $r s 8192678(1444 \mathrm{G}>\mathrm{A}) \mathrm{A}$-allele was associated with a $50 \%$ increase in the risk of micro/macroalbuminuria. The greatest risk was associated with the GA genotype (70\% compared to the GG genotype). Previous studies in Asian Indian [24] and Korean [25] samples have observed an autosomal dominant risk of nephropathy associated with the A-allele. We did not observe a greater risk associated with the AA genotype. One possible explanation may be that if risk was markedly increased in in AA subjects they may have experienced fatal CVD events. The subjects in our study sample were at least 10 years older and had a higher BMI than the Korean and Asian Indian studies. This is speculative but as described we observed that the AA genotype was associated with a higher proportion of small dense-LDL particles but we observed no association between genotype and plasma markers of oxidative stress. Small denseLDL particles are more susceptibility to oxidation [36], which may result in the generation of Ox-LDL, endothelial dysfunction, micro/macroalbuminuria [37] and increased CVD risk [38-41]. We also observed that there was greater ACE inhibitor and aspirin use in those with micro/macroalbuminuria compared to those without. These agents have anti-inflammatory and antioxidant properties [2] and this may account for the lack of association between genotype and the plasma markers of oxidative stress. There was no difference in statin use between those with or without micro/macroalbuminuria.

There are limitations to our study. We have chosen from the outset to examine one gene variant in $P G C-1 \alpha$ in relation to micro/macroalbuminuria and intermediate biochemical markers of oxidative stress. We have focussed on plasma TAOS and Ox-LDL as intermediate biochemical phenotypes. There are limitations to these measurements as discussed elsewhere $[32,42]$.
Further work should be performed to look at other variants within the $P G C-1 \alpha$ regulatory region to allow haplotype based analysis. This would also allow linkage disequilibrium across the region to be examined more fully. At first sight, the lack of any significant association between genotype and plasma markers of oxidative stress might appear to conflict with the associations observed for micro/macroalbuminuria and the proportion of small dense-LDL. One possible explanation for this might be the high proportion of aspirin and ACE inhibitor use described above. Furthermore, diabetes with increased obesity, oxidative burden, inflammation and hyperglycaemia might all overwhelm the $P G C-1 \alpha$ genotype 'strength of signal' and hence the genotype-intermediate phenotype association may not be observed. Case-control cross-sectional studies are prone to intrinsic bias, for example, due to possible altered rates of disease progression, subsequent progression of secondary phenotypes or genotype associations with death or treatment changes. Therefore, this SNP should be examined in a non-diabetic sample. Such influences are well-recognised confounders [43,44].

This report adds to the current literature relating to PGC-1 $\alpha$ gene variants. Further prospective analysis of the gene variant is required in diabetic and nondiabetic cohorts with measured plasma markers of oxidative stress, however access to such cohorts can be difficult [45].

\section{Acknowledgments}

Diabetes UK supported JWS (BDA: RD01/0001357) and the creation of UDACS. SEH is supported by the British Heart Foundation (RG2005 014).

\section{References}

[1] Cooke, J.P., The endothelium: a new target for therapy. Vascular Medicine. 2000; 5(1): 49-53. 
[2] Heitzer, T., et al., Endothelial dysfunction, oxidative stress, and risk of cardiovascular events in patients with coronary artery disease. Circulation. 2001; 104(22): 2673-8.

[3] Endemann, D.H. and Schiffrin, E.L. Endothelial dysfunction. J Am Soc Nephrol. 2004; 15(8): 1983-92.

[4] Rytand, D.A., The significance of albuminuria; proteinuria. Calif Med. 1949; 70(3): 174-6.

[5] Atkins, R.C., et al., Association between albuminuria and proteinuria in the general population: the AusDiab Study. Nephrol Dial Transplant. 2003; 18(10): 2170-4

[6] Navarro-Gonzalez, J.F. and Mora-Fernandez, C. The Role of Inflammatory Cytokines in Diabetic Nephropathy. J Am Soc Nephrol. 2008; 19(3): 433-442.

[7] Gall, M.A., et al., Albuminuria and poor glycemic control predict mortality in NIDDM. Diabetes. 1995; 44(11): 1303-9.

[8] Ek, J., et al., Mutation analysis of peroxisome proliferatoractivated receptor-gamma coactivator-1 (PGC-1) and relationships of identified amino acid polymorphisms to Type II diabetes mellitus. Diabetologia. 2001; 44(12): 2220-6.

[9] Choi, Y.S., et al., Impaired coactivator activity of the Gly482 variant of peroxisome proliferator-activated receptor gamma coactivator-1alpha (PGC-1alpha) on mitochondrial transcription factor A (Tfam) promoter. Biochem Biophys Res Commun. 2006; 344(3): 708-12.

[10] Rhee, J., et al., Regulation of hepatic fasting response by PPARgamma coactivator-1alpha (PGC-1): requirement for hepatocyte nuclear factor 4alpha in gluconeogenesis. Proc Natl Acad Sci USA. 2003; 100(7): 4012-7.

[11] Lai, C.Q., et al., PPARGC1A variation associated with DNA damage, diabetes, and cardiovascular diseases: the Boston Puerto Rican Health Study. Diabetes. 2008; 57(4): 809-16.

[12] Hara, K., et al., A genetic variation in the PGC-1 gene could confer insulin resistance and susceptibility to Type II diabetes. Diabetologia. 2002; 45(5): 740-3.

[13] Nitz, I., et al., Analysis of PGC-1alpha variants Gly482Ser and Thr612Met concerning their PPARgamma2-coactivation function. Biochem Biophys Res Commun. 2007; 353(2): 4816.

[14] Vimaleswaran, K.S., et al., Peroxisome proliferator-activated receptor-gamma co-activator-1alpha (PGC-1alpha) gene polymorphisms and their relationship to Type 2 diabetes in Asian Indians. Diabet Med. 2005; 22(11): 1516-21.

[15] Casteilla, L., M. Rigoulet and Penicaud, L. Mitochondrial ROS metabolism: modulation by uncoupling proteins. IUBMB Life. 2001; 52(3-5): 181-8.

[16] Negre-Salvayre, A., et al., A role for uncoupling protein-2 as a regulator of mitochondrial hydrogen peroxide generation. Faseb J. 1997; 11(10): 809-15.

[17] Dhamrait, S.S., et al., Cardiovascular risk in healthy men and markers of oxidative stress in diabetic men are associated with common variation in the gene for uncoupling protein 2. Eur Heart J. 2004; 25(6): 468-75.

[18] Cheurfa, N., et al., The Gly482Ser polymorphism in the peroxisome proliferator-activated receptor-gamma coactivator-1 gene is associated with hypertension in type 2 diabetic men. Diabetologia. 2004; 47(11): 1980-3.

[19] Ling, C., et al., Multiple environmental and genetic factors influence skeletal muscle PGC-1alpha and PGC-1beta gene expression in twins. J Clin Invest. 2004; 114(10): 1518-26.

[20] Andrulionyte, L., et al., Common polymorphisms of the PPAR-gamma2 (Pro12Ala) and PGC-1alpha (Gly482Ser) genes are associated with the conversion from impaired glucose tolerance to type 2 diabetes in the STOP-NIDDM trial. Diabetologia. 2004; 47(12): 2176-84.
[21] Fanelli, M., et al., The Gly482Ser missense mutation of the peroxisome proliferator-activated receptor gamma coactivator-1 alpha (PGC-1 alpha) gene associates with reduced insulin sensitivity in normal and glucose-intolerant obese subjects. Dis Markers. 2005; 21(4): 175-80.

[22] Deeb, S.S. and Brunzell, J.D. The Role of the PGC1alpha Gly482Ser Polymorphism in Weight Gain due to Intensive Diabetes Therapy. PPAR Res. 2009; 2009: 649286.

[23] Kunej, T., et al., A Gly482Ser polymorphism of the peroxisome proliferator-activated receptor-gamma coactivator-1 (PGC-1) gene is associated with type 2 diabetes in Caucasians. Folia Biol (Praha). 2004; 50(5): 157-8.

[24] Gayathri, S.B., et al., Association of the PPARGC1A gene polymorphism with diabetic nephropathy in an Asian Indian population (CURES-41). Metab Syndr Relat Disord. 8(2): 119-26.

[25] Jung L, S.J., Kim M., Chung K., Moon J.Y., Lee S., Lee T. and Lim C. The Polymorphisms of PPAR-gamma Coactivator 1alpha Gly482Ser (PGC-1alpha Gly482Ser) are Associated with the Nephropathy of Korean Patients with Type 2 Diabetes Mellitus. The Korean Journal of Nephrology. 2006; 25(5): 753-759.

[26] Stephens, J.W., et al., Cardiovascular risk and diabetes. Are the methods of risk prediction satisfactory? Eur J Cardiovasc Prev Rehabil. 2004; 11(6): 521-8.

[27] Alberti, K.G. and Zimmet, P.Z. New diagnostic criteria and classification of diabetes-again? Diabet Med. 1998; 15(7): 535-6.

[28] Amin, R., et al., Longitudinal relation between limited joint mobility, height, insulin-like growth factor 1 levels, and risk of developing microalbuminuria: the Oxford Regional Prospective Study. Arch Dis Child. 2005; 90(10): 1039-44.

[29] Amin, R., et al., The development of microalbuminuria is associated with raised longitudinal adiponectin levels in female but not male adolescent patients with type 1 diabetes. Diabetologia. 2008; 51(9): 1707-13.

[30] Magliano, D.J., et al., HPLC-detected albuminuria predicts mortality. J Am Soc Nephrol. 2007; 18(12): 3171-6.

[31] Sampson, M.J., et al., Plasma F2 isoprostanes: direct evidence of increased free radical damage during acute hyperglycemia in type 2 diabetes. Diabetes Care. 2002; 25(3): 537-41.

[32] Stephens, J.W., et al., Increased plasma markers of oxidative stress are associated with coronary heart disease in males with diabetes mellitus and with 10-year risk in a prospective sample of males. Clin Chem. 2006; 52(3): 446-52.

[33] Friedlander, Y., et al., Low density lipoprotein particle size and risk factors of insulin resistance syndrome. Atherosclerosis. 2000; 148(1): 141-9.

[34] Belo, L., et al., Changes in LDL size and HDL concentration in normal and preeclamptic pregnancies. Atherosclerosis. 2002; 162(2): 425-32.

[35] Schalkwijk, C.G., et al., Plasma concentration of C-reactive protein is increased in type I diabetic patients without clinical macroangiopathy and correlates with markers of endothelial dysfunction: evidence for chronic inflammation. Diabetologia. 1999; 42(3): 351-7.

[36] Hidaka, A., et al., Decrease in the particle size of low-density lipoprotein (LDL) by oxidation. Bioorg Med Chem Lett. 2005. 15(11): 2781-5.

[37] Ujihara, N., et al., Association between plasma oxidized lowdensity lipoprotein and diabetic nephropathy. Diabetes Res Clin Pract. 2002; 58(2): 109-14.

[38] Austin, M.A., et al., Low-Density Lipoprotein Subclass Patterns and Risk of Myocardial Infarction. JAMA: The Journal 
of the American Medical Association. 1988; 260(13): 19171921.

[39] Berneis, K.K. and Krauss, R.M. Metabolic origins and clinical significance of LDL heterogeneity. J Lipid Res. 2002; 43(9): 1363-79.

[40] Blake, G.J., et al., Low-density lipoprotein particle concentration and size as determined by nuclear magnetic resonance spectroscopy as predictors of cardiovascular disease in women. Circulation. 2002; 106(15): 1930-7.

[41] Vakkilainen, J., et al., Relationships between low-density lipoprotein particle size, plasma lipoproteins, and progression of coronary artery disease: the Diabetes Atherosclerosis Intervention Study (DAIS). Circulation 2003; 107(13): 1733-7.
[42] Stephens, J.W., M.P. Khanolkar and Bain, S.C. The biological relevance and measurement of plasma markers of oxidative stress in diabetes and cardiovascular disease. Atherosclerosis. 2009; 202(2): 321-9.

[43] Risch, N.J., Searching for genetic determinants in the new millennium. Nature. 2000; 405(6788): 847-56.

[44] Sing, C.F., J.H. Stengard and Kardia, S.L. Genes, environment, and cardiovascular disease. Arterioscler Thromb Vasc Biol. 2003; 23(7): 1190-6.

[45] Stephens, J.W., S.C. Bain and Humphries, S.E. Geneenvironment interaction and oxidative stress in cardiovascular disease. Atherosclerosis. 2008; 200(2): 229-38. 


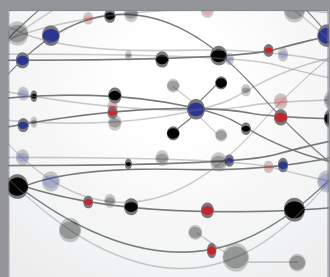

The Scientific World Journal
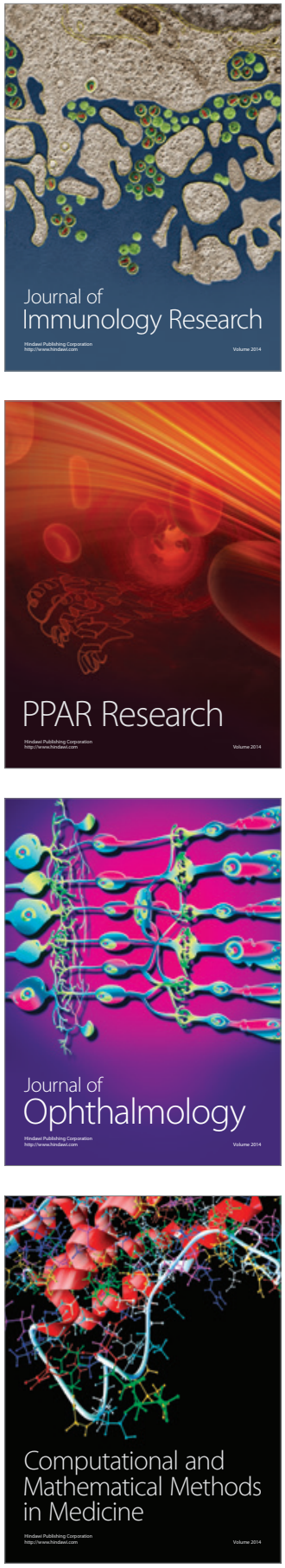

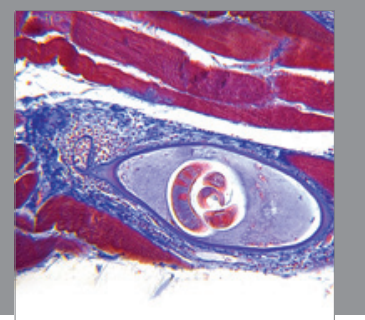

Gastroenterology

Research and Practice
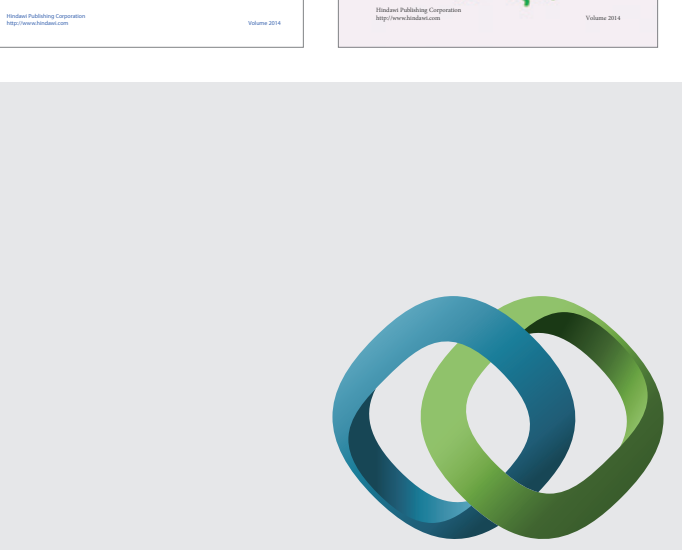

\section{Hindawi}

Submit your manuscripts at

http://www.hindawi.com
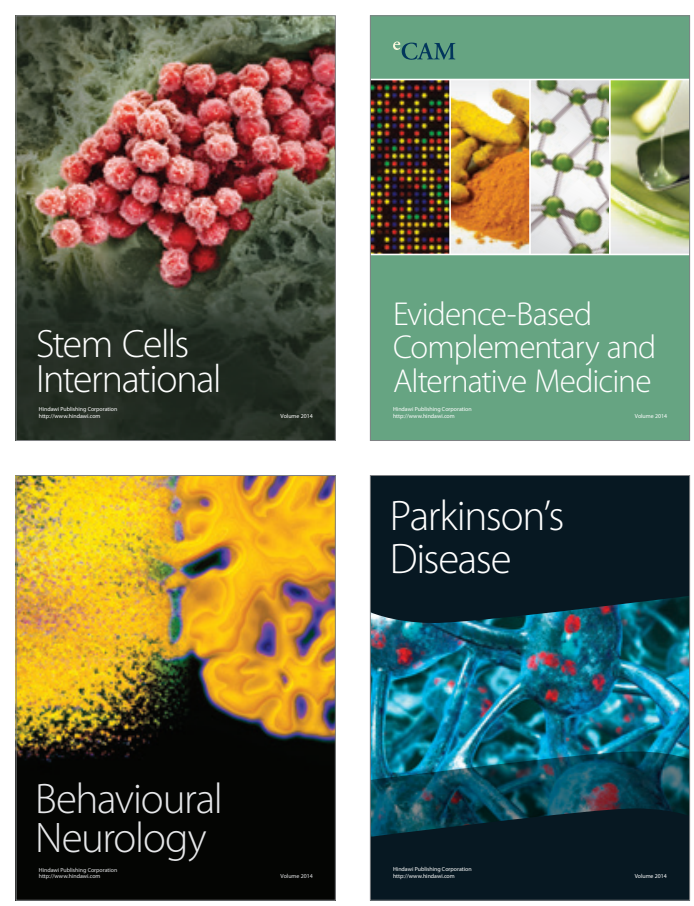

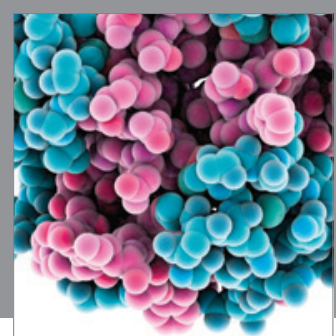

Journal of
Diabetes Research

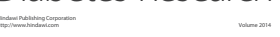

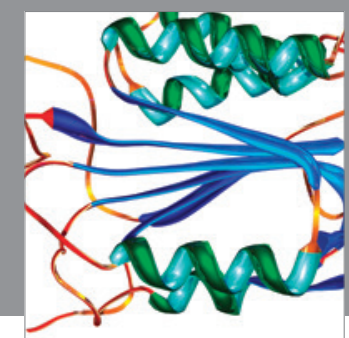

Disease Markers
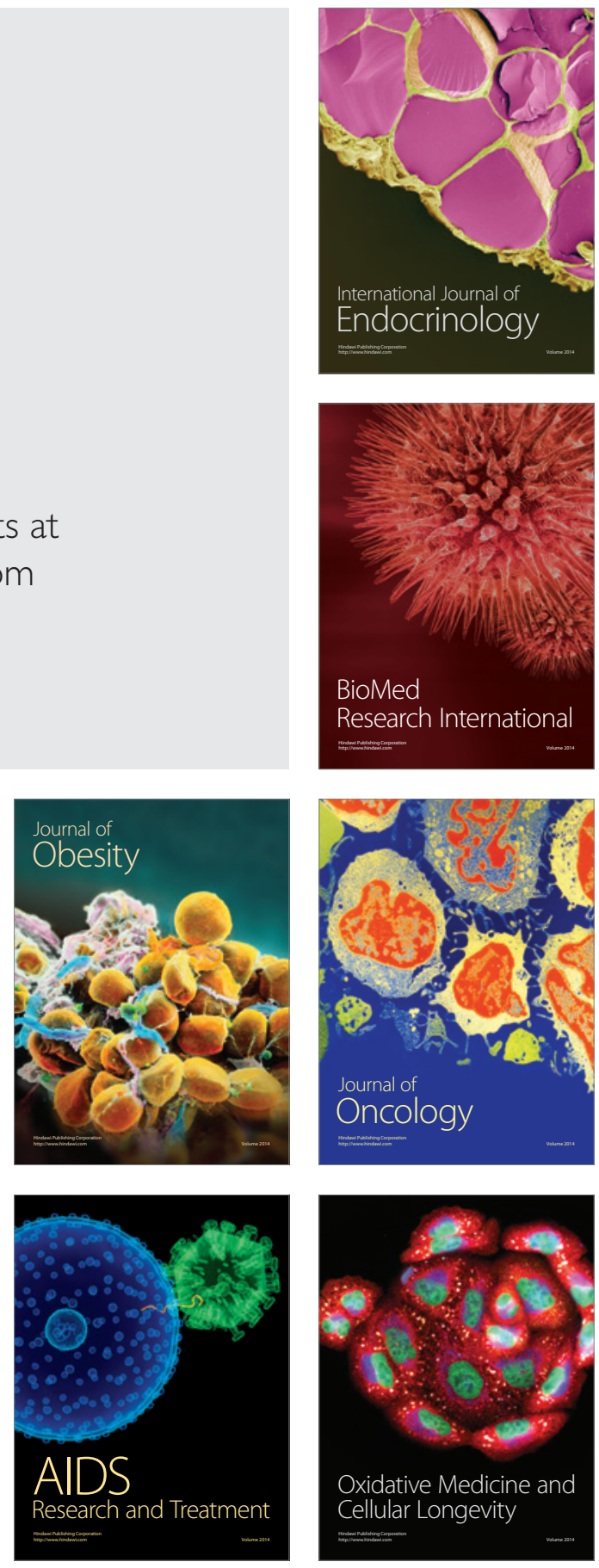